\title{
Impact of Intraoperative Gravity-Dependent Atelectasis Following Laparoscopic Liver Resection Performed in the Lateral Position
}

Ryoichi Miyamoto ( $\square$ goodfirst883@gmail.com )

Tsukuba Medical Center Hospital https://orcid.org/0000-0003-0687-6225

\section{Toshiro Ogura}

Saitama Cancer Center

Amane Takahashi

Saitama Cancer Center

Akifumi Kimura

Saitama Cancer Center

Shinichi Matsudaira

Saitama Cancer Center

Katsumi Amikura

Saitama Cancer Center

Yoshiyuki Kawashima

Saitama Cancer Center

\section{Research article}

Keywords: Laparoscopic liver resection, LLR, Morbidity, Short-term outcomes, Gravity-dependent atelectasis, GDA

Posted Date: July 9th, 2021

DOI: https://doi.org/10.21203/rs.3.rs-683021/v1

License: (1) (i) This work is licensed under a Creative Commons Attribution 4.0 International License. Read Full License 


\section{Abstract \\ Purpose}

Laparoscopic liver resection (LLR) is currently an accepted approach for liver surgery in select patients. The correlation between the intraoperative position and the presence of gravity-dependent atelectasis (GDA) has been well discussed. However, LLR is performed in the left half lateral position, and the relationship between this position and the presence of GDA remains unclear. We evaluated the extent to which the intraoperative left half lateral position affects the presence of GDA. Furthermore, univariate and multivariate analyses were performed to identify potential risk factors for LLR postoperative complications with a special emphasis on the presence of GDA by comparing various patient-, liver- and surgery-related factors in a retrospective cohort.

\section{Methods}

We retrospectively evaluated 129 patients who underwent LLR in the left half lateral position at the Saitama Cancer Center in Saitama, Japan between March 2011 and July 2020. The frequency and duration of GDA were investigated. We divided the cohort into with GDA and without GDA groups based on a cutoff value ( $\geq 5$ days, $n=61$ and $<5$ days, $n=68$, respectively). Using multivariate analysis, the duration of GDA and several risk factors for LLR postoperative complications were independently assessed.

\section{Results}

Postoperative GDA was observed in 61 patients (47\%) and lasted for 1 to 8 days in these patients. The mean duration of GDA was 4.3 days. Multivariate logistic regression analysis revealed a GDA duration of 5 days or more (odds ratio [OR], 2.03; $p=0.001)$ and an operating time $>388$ minutes $(O R, 5.31 ; p<0.001)$ to be independent risk factors for LLR postoperative complications.

\section{Conclusions}

The incidence and duration of postoperative GDA are considered useful predictors of postoperative complications, and these predictors should be assessed to improve the short-term outcomes of patients undergoing LLR.

\section{Introduction}

Laparoscopic liver resection (LLR) for benign tumors was initially reported by Reich et al. in 1991 and is now used to treat primary and metastatic malignant tumors [1-3]. Initially, laparoscopic procedure was not widely accepted due to the difficulty in controlling bleeding. Improvements in laparoscopic techniques 
and instruments, as well as accumulated experience have led to a wider acceptance of LLR by trained surgeons $[4,5]$. The international position on LLR was addressed by experts in hepatobiliary surgery at an international consensus conference in Louisville, Kentucky (USA) in November 2008 [6]. The experts concluded that LLR is considered as a safe and effective approach when carried out by experienced surgeons [6].

Recent studies have suggested that LLR leads to fewer postoperative complications such as operative blood loss and a shorter duration of hospital stay than does conventional open liver surgery [3,7-9]. Simillis et al. reported that the volume of operative blood loss and duration of hospital stay were significantly reduced after laparoscopic surgery [3]. Lesurtel et al. also reported that the laparoscopic approach was associated with less blood loss and the absence of specific postoperative complications of hepatic resection [10].

Regarding these complications, the relationships between postoperative thoracic complications and laparoscopic surgery have been well discussed. Previous studies have reported that thoracic complications are associated with longer operative times, an increased number of operative ports, larger values of positive end-tidal $\mathrm{CO}_{2}$, and intraoperative positions $[11,12]$. In terms of thoracic complications and the intraoperative position, Craig et al initially reported that $30 \%$ of patients who underwent thoracic surgery in the lateral position exhibited gravity-dependent atelectasis (GDA) in the contralateral lung [13]. Zhao et al. also reported that $47 \%$ of patients who underwent laparoscopic urological surgery in the lateral position exhibited GDA in the contralateral lung [11]. In terms of LLR, which is performed in the left half lateral position, the relationship between this intraoperative position and the presence of GDA remains unclear.

In this study, we evaluated the extent to which the intraoperative left half lateral position affects the presence of GDA. Furthermore, univariate and multivariate analyses were performed to identify potential risk factors for LLR postoperative complications with a special emphasis on the presence of GDA by comparing various patient-, liver- and surgery-related factors in a retrospective cohort.

\section{Materials And Methods}

\section{Patients}

We retrospectively evaluated 217 consecutive patients who underwent LLR at the Saitama Cancer Center in Saitama, Japan between March 2011 and July 2020. The ethics committee of the Saitama Cancer Center approved this study (\#1115). First, we excluded 21 patients who underwent surgeries that were more extensive standard LLR procedures, such as simultaneous colectomy. Second, 67 patients who underwent LLR in the supine position were excluded. A total of 129 patients who underwent LLR in the left half lateral position were included in the final cohort.

\section{Definition of the presence of GDA}


We routinely performed postoperative plain chest radiography immediately after the operation and on postoperative days 1, 2, 3, 5 and 7. The presence of GDA was defined as evidence of atelectasis or infiltration in the left lung on plain chest radiographs taken immediately after the operation with respect to the preoperative radiographs (Fig. 1A and 1B). Similarly, an improvement in GDA was also recorded when there was no evidence of atelectasis or infiltration in the left lung on the plain chest radiographs. The frequency and duration of GDA were investigated. For the129 patients who underwent LLR in the left half lateral position, the following patient characteristics were compared between the patients with or without GDA: age, sex ratio, body mass index (BMI), American Society of Anesthesiologists (ASA) score, history of respiratory comorbidities, history of diabetes mellitus, history of abdominal surgery, liver fibrosis, tumor size, number of lesions, tumor characteristics, surgical procedure, operating time, intraoperative blood loss, length of postoperative hospital stay and postoperative complications. Liver fibrosis was graded according to the Metavir score [14]. The postoperative complications were also graded according to the Clavien-Dindo classification system [15].

\section{Patient-, liver- and surgery-related risk factors for short-term outcomes}

Univariate and multivariate analyses were performed to not only assess the duration of GDA but also identify potential risk factors for LLR postoperative complications by comparing various patient-, liverand surgery-related factors. Cutoff values for each continuous variable were defined according to the ROC curve analysis results.

For patient-related risk factors, we focused on an older age, the male sex, a high BMI, a high ASA score, a history of respiratory comorbidities, a history of diabetes mellitus and a history of abdominal surgery.

For liver-related factors, we collected data regarding tumor size (>31 mm), multiple lesions, liver fibrosis (Metavir score F1 to F4) and malignant pathology.

For surgery-related factors, we focused on surgical procedure, operating time ( $>388$ minutes), and intraoperative blood loss (>295 grams).

ROC curves visually represent the sensitivity (i.e., the probability of correctly identifying an event such as death) and specificity (i.e., the probability of correctly identifying a nonevent) of various cutoff values.

\section{Surgical procedures and perioperative management}

We routinely performed the laparoscopic technique with five ports, a 10-mmHg pneumoperitoneum and a flexible angle laparoscope. The hand-assisted technique was not used. Pringle's maneuver was routinely used [16]. Parenchymal liver transection was performed by the THUNDERBEAT ${ }^{\text {TM }}$ system (TS) (Olympus Medical Systems Corp., Tokyo, Japan) and the Cavitron ultrasonic surgical aspirator (CUSA: Integra Lifesciences Corporation, NJ, USA). Bipolar coagulation was used to treat minor bleeding. The resected liver was placed in a plastic bag and extracted, without fragmentation, through a small abdominal incision. The drainage tube was routinely placed on the resected liver surface. The methods of managing 
the drain and checking the total bilirubin levels in the drainage fluid were standardized in this study. The drain was removed if the drainage fluid was clear and both bile leakage and bacterial contamination were absent. All patients received prophylactic antibiotics either intraoperatively or for 1 or 2 days postoperatively.

\section{Statistical analyses}

Correlations with patient background data were analyzed using a $\chi^{2}$ test or Fisher's exact test, as appropriate. For the multivariate analysis, a multiple logistic regression analysis yielding odds ratios and 95\% confidence intervals (Cls) was used to identify risk factors for LLR postoperative complications (with $p$ values $<0.05$ ). Statistical analyses were performed using a statistical analysis software package (SPSS Statistics, version 21; IBM, Armonk, NY, USA), and $p$ values $<0.05$ were considered significant.

\section{Result}

\section{Patient characteristics}

The backgrounds of the patients either with or without GDA in the left half lateral position are presented in Table 1. Significant differences were observed between the two groups with regard to BMI $(p=0.031)$, a history of respiratory comorbidities $(p=0.001)$, a history of diabetes mellitus $(p<0.001)$, operating time $(p=0.025)$, and postoperative complications $(p=0.001)$.

Regarding the postoperative complications in the patients with GDA, there were 4 patients with mild-tosevere pneumonia, 2 patients with bile leakage and 1 patient with surgical site infection. Similarly, among the patients without GDA, there was 1 patient with bile leakage and 1 patient with surgical site infection.

\section{Frequency and duration of GDA}

In the present cohort, postoperative GDA was observed in 61 patients (47\%). In these 61 patients, the duration of GDA ranged from 1 to 8 days. The values of the duration of GDA were normally distributed. The mean duration of GDA was 4.3 days. Therefore, 5 days was used as the cutoff value in the present study.

Univariate analyses of risk factors for short-term outcomes during different treatment phases (Table 2)

There was a significant difference in the duration with GDA with respect to the incidence of postoperative complications (high, 4 (44\%) vs. low, $3(5.7 \%), p<0.001)$.

In terms of the patient-related risk factors, BMI (high, $5(15 \%)$ vs. low, $2(6.8 \%), p=0.045)$, a history of respiratory comorbidities (yes, $2(25 \%)$ vs. no, $5(10 \%), p=0.035)$, and a history of diabetes mellitus (yes, $4(17 \%)$ vs. no, $3(7.8 \%), p=0.015)$ significantly affected the incidence of postoperative complications.

There were no parameters significantly affected the incidence of postoperative complications with respect to liver-related risk factors. 
In terms of surgery-related risk factors, operating time (> 388 minutes, $6(19 \%)$ vs. < 388 minutes, 1 (3.3\%), $p=0.001)$ significantly affected the incidence of postoperative complications.

\section{Multivariate analysis (Table 3)}

Multivariate logistic regression analysis revealed a GDA duration of 5 days or more (odds ratio [OR], 2.03; $p=0.001)$ and an operating time $>388$ minutes $(O R, 5.31 ; p<0.001)$ to be independent risk factors for LLR postoperative complications.

\section{Discussion}

In the present study, postoperative GDA was observed in 61 patients (47\%), and the mean duration of GDA was 4.3 days (1-8 days). Furthermore, the multivariate analysis results indicated that the incidence of postoperative GDA correlated with the postoperative outcomes of the patients who underwent LLR in the left half lateral position. Thus, the incidence and duration of postoperative GDA are assumed to be useful predictors for postoperative complications, and these predictors should be assessed to improve the short-term outcomes of patients undergoing LLR.

In terms of the mechanism that causes GDA after surgery in the lateral position, Rheder et al was the first to suggest that there is a preferential distribution of tidal volume to the nondependent lung and preferential ventilation of the dependent lung in the lateral position [17]. Furthermore, it has also been reported that not only increase in abdominal pressure by laparoscopic surgery but also relaxation of the diaphragm with the use of muscle relaxants elevate the diaphragm, further decreasing the functional residual capacity of the lower lungs [17]. Regarding the increase in abdominal pressure, it is suggested that the pneumoperitoneum pressure in laparoscopic surgery may further raise the diaphragm and cause ventilation disorders. Furthermore, Kaneko et al showed that in subjects in the lateral position at lung volumes of less than $50 \%$ of total lung capacity, the nondependent regions of the lung receive a relatively large proportion of the inspired volume [18].

Long-term outcomes of LLR have been reported in some previous studies $[7,19,20]$. The results of these studies revealed that LLR was comparable to conventional open liver resection in terms of resection margins, recurrence rates, and long-term outcomes. However, the short-term outcomes of LLR have hardly been discussed until now. The reason for the lack of studies on short-term outcomes and risk factors for postoperative complications is that strict patient selection criteria for LLR were established by an international consensus on LLR [6]. Tranchart et al first reported that the postoperative complication rate increases by $60 \%$ with each additional increment of time required for LLR [7]. The potential relationship between operating time and risk of short-term outcomes was initially reported in 1960 [21,22]. Nobili et al reported that operating time was identified by multivariate analysis as an independent risk factor for pulmonary and infectious complications after open hepatic resection [23].

According to our multivariate analysis, in addition to operating time, a GDA duration of 5 days was revealed to be an independent risk factor for LLR postoperative complications. Questions regarding the 
mechanisms by which the incidence of postoperative GDA potentially affects the postoperative outcomes of patients who undergo LLR remain unanswered. One potential hypothesis is related to the incidence rate of GDA being $47 \%$ and most of the cases having improved spontaneously by the 4 th postoperative day. Conversely, it is suggested that patients with GDA lasting more than 5 days may develop severe pneumonia. In fact, mild-to-severe pneumonia was observed in all 4 patients with moderate or severe complications (Clavien-Dindo classification II to IV) in the present study. Furthermore, diabetes mellitus has been reported in the literature to be a risk factor for infectious complications, including pneumonia, after hepatic resection $[23,24]$. Indeed, the present study showed that the proportion of patients with diabetes mellitus was higher in the with GDA group than in the without GDA group.

In conclusion, we found that a GDA duration of 5 days or more may be a useful predictor of postoperative complications, and this predictor should be assessed improve the short-term outcomes of patients undergoing LLR. Therefore, we assumed that the early termination of bed rest, including the start of respiratory rehabilitation, and early improvements in GDA are crucial for the prevention of complications, including pneumonia. The present study had limitations; it was a retrospective single-center study with a relatively small sample size. These results require confirmation by additional multicenter large-scale studies and prospective randomized controlled studies.

\section{Declarations}

\section{Ethics approval and consent to participate}

All procedures performed in studies involving human participants were in accordance with the ethical standards of the institutional research committee (\#2020-1115) and with the 1964 Helsinki declaration and its later amendments or comparable ethical standards. Informed consent was obtained from all individual participants included in the study.

\section{Consent to publish}

Informed consent was obtained from all individual participants included in the study.

\section{Availability of data and materials}

The datasets used and analyzed during the current study available from the corresponding author on reasonable request.

\section{Competing interests}

The authors declare that they have no conflict of interest.

\section{Funding}

This study had no funding. 
Authors' Contributions

MR and OT collected, analyses the data. MR wrote the manuscript, prepared the tables and figures. All authors read and approved the final manuscript.

\section{Acknowledgements}

We have no acknowledgments to make.

\section{References}

1. Reich H, McGlynn F, Decaprio J, Budin R. Laparoscopic excision of benign liver lesions. Obstet Gynecol. 1991;78:956-8.

2. Cherqui D, Laurent A, Tayar C, Chang S, Van Nhieu JT, Loriau J, Loriau J, Karoui M, Duvoux C, Dhumeaux D, Fagniez P. Laparoscopic liver resection for peripheral hepatocellular carcinoma in patients with chronic liver disease: midterm results and perspectives. Ann Surg. 2006;243:499-506.

3. Simillis C, Constantinides VA, Tekkis PP, Darzi A, Loveqrove R, Jiao L, Anthony A. Laparoscopic versus open hepatic resections for benign and malignant neoplasms-a meta-analysis. Surgery. 2007;141:203-11.

4. Imura S, Shimada M, Utsunomiya T, Morine Y, Wakabayashi G, Kaneko H. Current status of laparoscopic liver surgery in Japan: results of a multicenter Japanese experience. Surg Today. 2014;44:1214-9.

5. Ban D, Kudo A, Ito H, Mitsunori Y, Matsumura S, Aihara A, Ochiai T, Tanaka S, Tanabe M, Itano O, Kaneko $\mathrm{H}$, Wakabayashi $\mathrm{G}$. The difficulty of laparoscopic liver resection. Updates Surg. 2015;67:123-8.

6. Buell JF, Cherqui D, Geller DA, O’Rourke N, lannitti D, Dagher I, Koffron AJ, Thomas M, Gayet B, Han HS, Wakabayashi G, Belli G, Kaneko H, Ker CG, Scatton O, Laurent A, Abdalla EK, Chaudhury P, Dutson E, Gamblin C, D'Angelica M, Nagorney D, Testa G, Labow D, Manas D, Poon RT, Nelson H, Martin R, Clary B, Pinson WC, Martinie J, Vauthey JN, Goldstein R, Ro-ayaie S, Barlet D, Espat J, Abecassis M, Rees M, Fong Y, McMasters KM, Broelsch C, Busuttil R, Belghiti J, Strasberg S, Chari RS. The international position on laparoscopic liver surgery: The Louisville Statement, 2008. Ann Surg. 2009;250:825-30.

7. Tranchart H, Di Giuro G, Lainas P, Roudie J, Agostini H, Franco D, Dagher I. Laparoscopic resection for hepatocellular carcinoma: a matched-pair comparative study. Surg Endosc. 2010;24:1170-6.

8. Belli G, Fantini C, D’Agostino A, Cioffi L, Langella S, Russolillo N, Belli A. Laparoscopic versus open liver resection for hepatocellular carcinoma in patients with histologically proven cirrhosis: short- and middle-term results. Surg Endosc. 2007;21:2004-11.

9. Kaneko H, Takagi S, Otsuka Y, Tsuchiya M, Tamura A, Katagiri T, Maeda T, Shiba T. Laparoscopic liver resection of hepatocellular carcinoma. Am J Surg. 2005;189:190-4. 
10. Lesurtel M, Cherqui M, Laurent A, Tayar C, Fagniez P. Laparoscopic versus open left lateral hepatic lobectomy: a case-control study. J Am Coll Surg. 2003;196:236-42.

11. Zhao L, Han J, Loeb S, Tenggardjaja C, Rubenstein R, Smith N, Nadler R. Thoracic complications of urologic laparoscopy: correlation between radiographic findings and clinical manifestations. J Endourol. 2008;22:607-14.

12. Murdock CM, Wolff AJ, Geem TV. Risk factors for hypercarbia, subcutaneous emphysema, pneumothorax, and pneumomediastinum during laparoscopy. Obstet Gynecol. 2000;95:704-9.

13. Craig JOC, Bromley LL, Williams R. Thoracotomy and the contralateral lung. A study of the changes occurring in the dependent and contralateral lung during and after thoracotomy in lateral decubitus. Thorax. 1962;17:9-15.

14. Goodman ZD. Grading and staging systems for inflammation and fibrosis in chronic liver diseases. J Hepatol. 2007;47:598-607.

15. Dindo D, Demartines N, Clavien PA. Classification of surgical complications: a new proposal with evaluation in a cohort of 6336 patients and results of a survey. Ann Surg. 2004;240:205-13.

16. Di Giuro G, Lainas P, Franco D, Dagher I. Laparoscopic left hepatectomy with prior vascular control. Surg Endosc. 2010;24:697-9.

17. Rehder K, Hatch DJ, Sessler AD, Fowler WS. The function of each lung of anesthetized and paralyzed man during mechanical ventilation. Anesthesiology. 1972;37:16-26.

18. Kaneko K, Milic-Emili J, Dolovich MB, Dawson A, Bates DV. Regional distribution of ventilation and perfusion as a function of body position. J Appl Physiol. 1966;21:767-77.

19. Kazaryan AM, Marangos IP, Rosok BI, Rosseland AR, Villanger O, Fosse E, Mathisen O, Edwin B. Laparoscopic resection of colorectal liver metastases: surgical and long-term oncologic outcome. Ann Surg. 2010;252:1005-12.

20. Castaing D, Vibert E, Ricca L, Azoulay D, Adam R, Gayet B. Oncologic results of laparoscopic versus open hepatectomy for colorectal liver metastases in two specialized centers. Ann Surg. 2009;250:849-55.

21. Berard F, Gandon J. Postoperative wound infections: the influence of ultraviolet irradiation of the operating room and of various other factors. Ann Surg. 1964;160:1-192.

22. PHLS. Incidence of surgical wound infection in England and Wales: A report of the Public Health Laboratory Service. Lancet. 1960;2:659-653.

23. Nobili C, Marzano E, Oussoultzoglou E, Rosso E, Addeo P, Bachellier P, Jaeck D, Pessaux P. Multivariate analysis of risk factors for pulmonary complications after hepatic resection. Ann Surg. 2012;255:540-50.

24. Okabayashi T, Nishimori I, Yamashita K, Sugimoto T, Yatabe T, Maeda H, Kobayashi M, Hanazakiet K. Risk factors and predictors for surgical site infection after hepatic resection. J Hosp Infect. 2009;73:47-53. 


\section{Tables}

Due to technical limitations, table 1, 2, 3 is only available as a download in the Supplemental Files section.

\section{Figures}
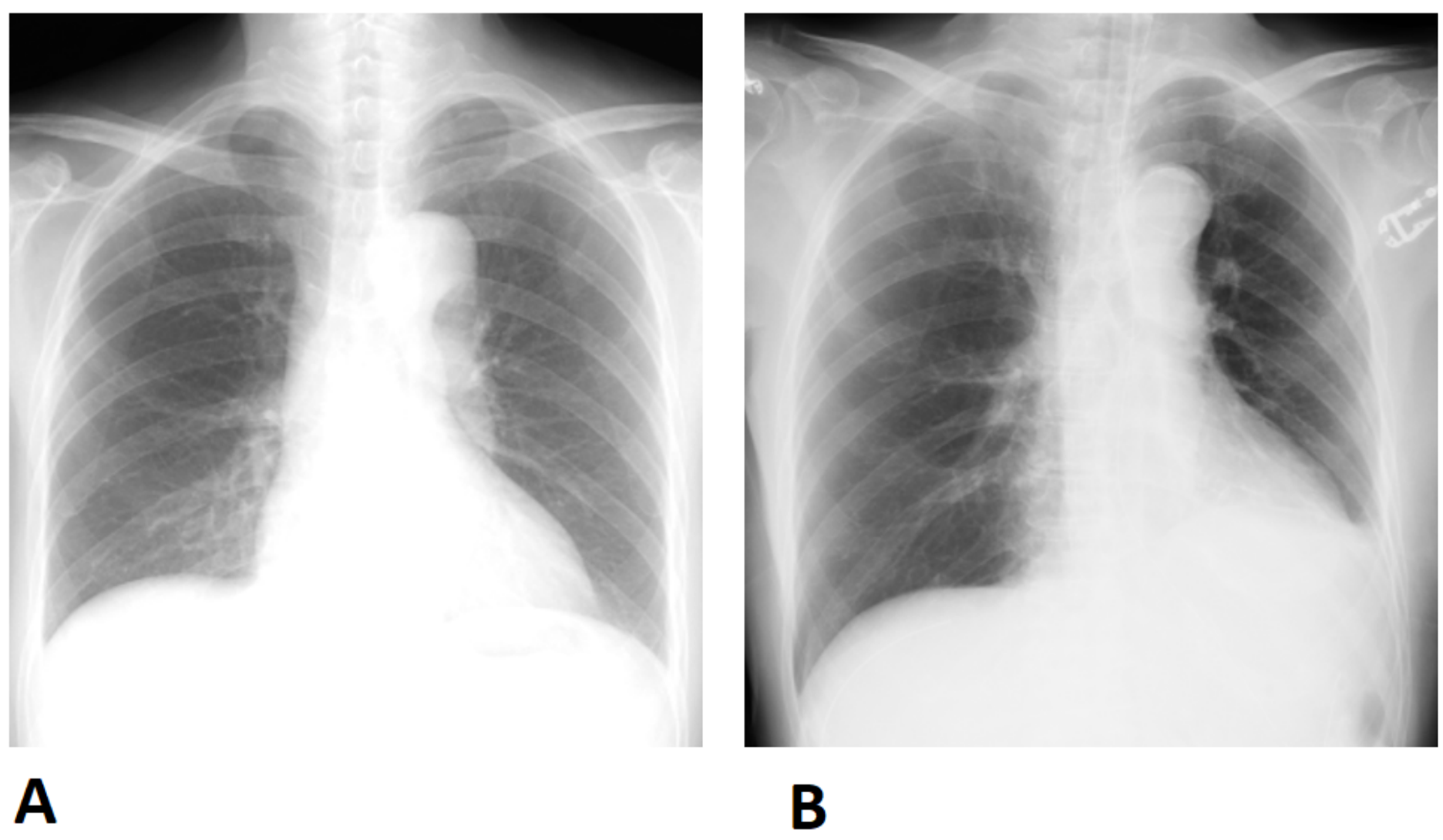

B

\section{Figure 1}

A A preoperative plain chest radiograph is shown. B A postoperative plain chest radiograph taken immediately after the operation is shown. This image shows an elevated diaphragm, with atelectasis or infiltration in the left lung, with respect to the preoperative findings. Therefore, this case was defined as positive for GDA.

\section{Supplementary Files}

This is a list of supplementary files associated with this preprint. Click to download.

- Table1.docx

- Table2.docx

- Table3.docx 